\title{
SCALAR FORMULATION \\ OF IDEAL CHARGED GAS FLOW
}

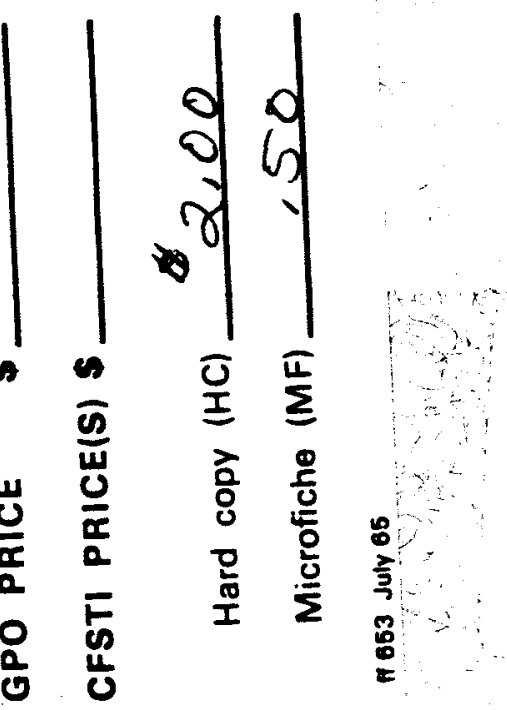

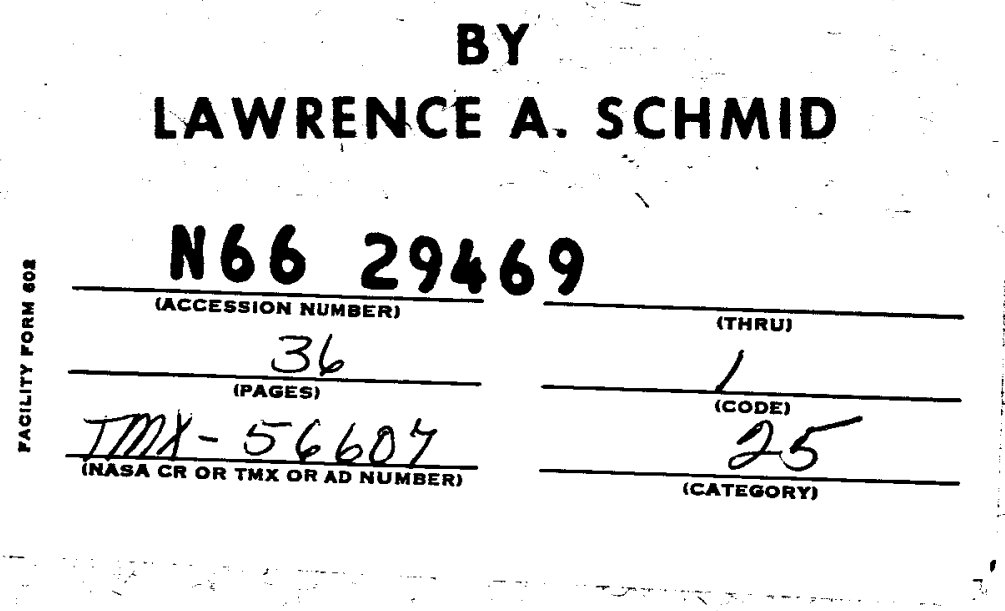

\section{JUNE 1965}

GODDARD SPACE FLIGHT CENTER GREENBELT, MARYLAND

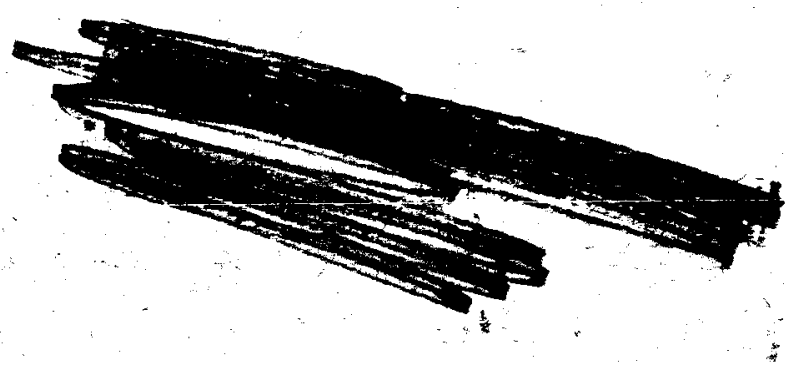


SCALAR FORMULATION OF IDEAL CHARGED GAS FLOW

by

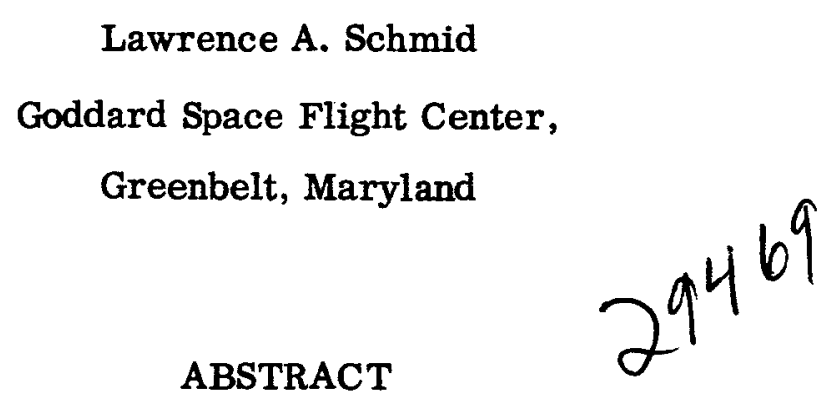

In the case of adiabatic flow of an ideal electron or ion gas in the presence of gravitational and electromagnetic fields, it is possible to derive a system of scalar equations of motion for the gas that provides an alternative to the Euler formulation. The new dynamical variables that replace the velocity are three scalars, one of which is a generalizatic a of the flow potential and satisfies a generalized Hamilton-Jacobi equation. The other two are constants of motion that specify the intrinsic $v$. ticity, which is that part of the total vorticity that is not produced by the external fields, but rather is simply a residue of the initial conditions of the gas. It is this intrinsic vorticity that satisfies the generalization of the simple vorticity conservation theorem. The same formalism can be adapted to the case of constant-temperature flow, and to the flow of an arbitrary barotropic fluid. 


\section{SCALAR FORMULATION OF IDEAL CHARGED GAS FLOW}

\section{INTRODUCTION}

Euler's equation for an ideal electrically uncharged fluid, which is a vector equation, can be reduced to a scalar equation in the case of potential flow. The conditions that must be fulfilled in order for potential flow to exist are the following: (1) The pressure must be a function of the fluid density alone. (2) The external force acting on the fluid must be the gradient of a scalar potential. (3) The curl of the fluid velocity must everywhere vanish.

According to the Helmholtz (or Kelvin) vorticity conservation theorem, if the third condition is satisfied at any given instant of time, it will continue to hold for all time.

When the above conditions are satisfied, the velocity can be expressed as the gradient of a flow potential :

$$
v_{n}=\nabla_{n} S
$$

The most important special case for which the first of the above conditions is satisfied is that of isentropic flow, for which the specific entropy is everywhere constant for all time. For this case, Euler's equation becomes

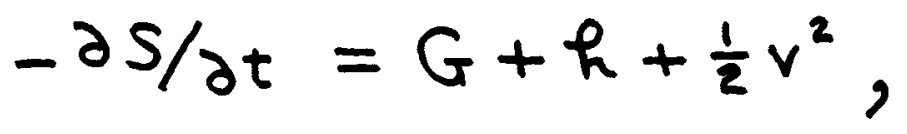


where $G$ is the potential for the external force (which throughout will be taken to be gravitation), and $\mathcal{h}$ is the specific enthalpy。

In the case of time-independent flow, $-\partial S / \partial t$ reduces to a constant $E$ having the dimensions of energy/unit mass, and (1.2) becomes

$$
E=G+h+\frac{1}{2} v^{2},
$$

which is Bernoulli's equation.

It is interesting to note that

$$
D S=\frac{1}{2} v^{2}-(G+h),
$$

where

$$
D=\frac{\partial}{\partial t}+v \cdot \nabla
$$

is the substantial time derivative. But from single-particle Hamilton-Jacobi theory we have the following relations:

$$
\nabla d=m v
$$

and

$$
D \&=m\left(\frac{1}{2} v^{2}-G\right)=L,
$$


where $\mathcal{L}$ is Hamilton's Principal Function, $m$ is the particle mass, and $L$ is the particle Lagrangian. Comparison of equations (1.6) with $(1.1)$ and $(1.4)$ indicates that the flow potential $S$ plays a role analogous to $\mathrm{d} / \mathrm{m}$. Comparison of the potential energy terms in (1.4) and (1.6b) indicates that, as far as dynamical effects are concerned, the enthalpy $h$ plays the role of a thermal potential which is to be treated in the same way as the gravitational potential $\mathbf{G}$.

This analogy between the formulation of potential flow in terms of the flow potential $\mathrm{S}$ and the single-particle Hamilton-Jacobi formalism is further reinforced by using (1.1) to eliminate $v^{2}$ in (1.2). The resulting nonlinear particle differential equation for $S_{\text {has }}$ the form of the Hamilton-Jacobi equation:

$$
-\partial S / \partial t=G+h+\frac{1}{2}(\nabla S)^{2}
$$

This equation provides a scalar alternative to Euler's equation in the case of irrotational, isentropic flow. Once (1.7) has been solved (for given $\mathbf{G}$ and $\boldsymbol{h}$ ), $\mathbf{y}$ can be found from (1.1) and the fluid particle density $P$ can be found from the continuity equation as follows:

$$
D \ln \rho=-\nabla \cdot v=-\nabla^{2} S \text {. }
$$

It would be desirable to generalize this scalar formulation in three respects: (1) by replacing the isentropic requirement that the entropy be everywhere constant by the weaker adiabatic requirement that the entropy be constant along any given flow line, in which case the pressure will be a function of the entropy as well as the density; (2) 
by including the non-potential electromagnetic forces that act on an electrically charged fluid; (3) by including rotational as well as irrotational flow. This generalization will be carried out in the work that follows. We shall see, moreover, that a slight modification of the formalism suffices to include the constant temperature case for which the temperature must be constant on a given flow line (but may vary from line to line), and the case of an arbitrary barotropic fluid for which the pressure is an arbitrary function of the pressure alone.

Throughout the derivation we shall consider only an ideal charged fluid. In the case of an electron gas, for example, this means that we shall neglect not only its selfviscosity, but also the frictional drag between it and the ion gas which necessarily occupies the same region of space.

\section{EULER FORMULATION}

Our starting point is the familiar Euler equation for an ideal charged fluid, such as an electron or ion gas in which viscosity and heat conduction are ignored, in the presence of a gravitational potential $G$ and electric and magnetic fields $\underset{m}{\mathbf{E}}$ and $\mathbf{B}$ :

$$
m D v=-m \nabla g-e^{-1} \nabla_{m} P+q E+(q / c) \not \times m
$$

where $m$ and $q$ are the particle mass and charge, $P$ the pressure, and $\boldsymbol{P}$ the particle density.

This equation must be supplemented by the continuity equation: 


\section{$\partial e / \partial t+\nabla_{m} \cdot(e v)=0$}

or

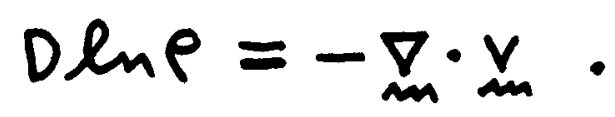

These two equations must be further supplemented by an equation that specifies the nature of the thermodynamic constraint on the flow. In the case of isentropic flow of a perfect gas for which the ratio of specific heats is $\gamma$, this thermodynamic sidecondition is

$$
P / \rho^{r}=\text { constant. (Isentropic Flow) }
$$

For the weaker requirement that the flow be adiabatic, i.e. that the specific entropy be constant along a flow-line, the thermodynamic equation is

$$
D \ln P=\gamma D \ln \rho=-\gamma \nabla \cdot{ }_{m} .
$$

(Adiabatic Flow)

A necessary first step in deriving a scalar alternative to Euler's equation is to eliminate $P$ from (2.1). This is accomplished by means of the following thermodynamic identity:

$$
\rho^{-1} \nabla_{m} P=m T \nabla_{m} \Delta-m \nabla_{m} h
$$

where $T$ is the temperature, and $\Delta$ and $\mathcal{h}$ are the specific entropy and enthalpy respectively. 
We shall also eliminate the electric and magnetic field intensities from (2.1) in favor of the vector and scalar potentials $A$ and $A^{\circ}$ by means of the familiar relations

$$
E_{m}=-\nabla A^{0}-\partial A / c \partial t
$$

and

$$
\mathbb{m}_{m}=\nabla_{m} \times \mathrm{A}_{m}
$$

Using (2.5) and (2.6), (2.1) can be cast into the following form:

$$
\begin{aligned}
\frac{\partial}{\partial t}\left(x_{m}+\frac{q}{m c} A\right)= & -\nabla\left(G+h+\frac{1}{2} v^{2}+\frac{q}{m} A^{0}\right) \\
& +T \nabla_{m} A+v_{m} \times\left[\left(\nabla_{m} \times x\right)+\frac{q}{m c} B\right] .
\end{aligned}
$$

The Thermal Function

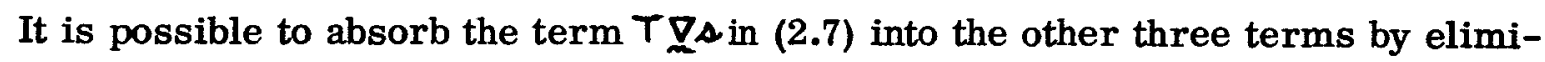
nating the temperature $T$ in favor of a scalar function $\widetilde{\mathcal{J}}$, called the thermal function, that is defined by the following equation:

$$
D \mathfrak{F}=T
$$

The utility of the thermal function follows from the identity

$$
\begin{aligned}
(D \mathcal{J})(\nabla s)= & (D \Delta)(\nabla \mathfrak{J})-\partial(\Delta \nabla \mathfrak{J}) / \partial t \\
& +\nabla_{m}(\Delta \partial \mathfrak{J} / \partial t)+\Sigma_{m} \times(\nabla \Delta \times \nabla \mathfrak{J}) .
\end{aligned}
$$

We now formally introduce the requirement that the flow be adiabatic:

$$
D A=0 \text {. }
$$


Using (2.8) and (2.10) in (2.9), we arrive at the desired expression for T⿱⿴囗十心

$$
\begin{aligned}
T \nabla_{m}= & -\partial(\Delta \nabla \mathcal{J}) / \partial t+\nabla(\Delta \partial \mathcal{J} / \partial t) \\
& +v_{m} \times(\nabla s \times \nabla J) .
\end{aligned}
$$

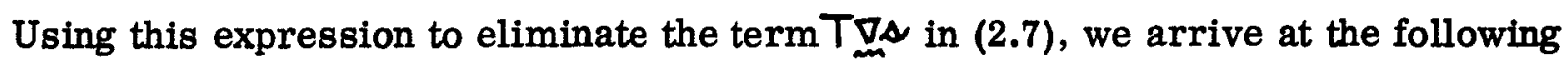
form of Euler's equation:

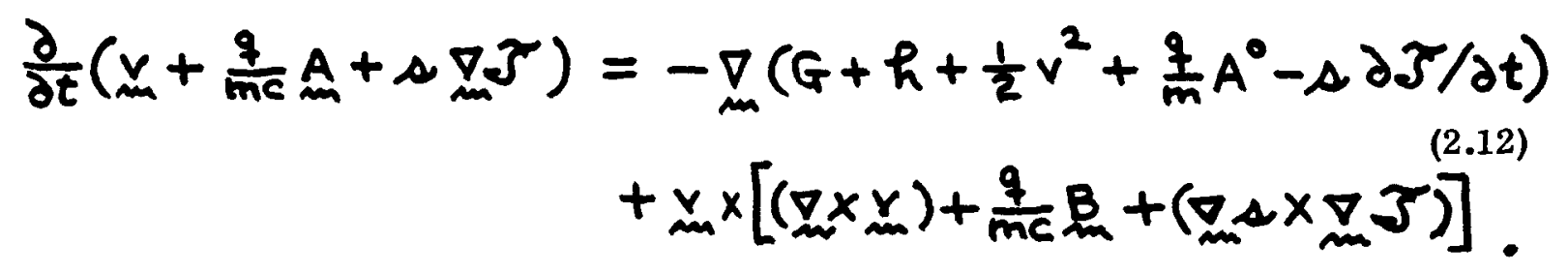

Before pursuing the formal development further, it would be well to discuss the physical significance of the thermal function $\widetilde{\mathcal{J}}$. From (2.8) we note that $\widetilde{\mathcal{F}}$ could be regarded as the reading of a clock that moves with the fluid and whose rate is proportional to the temperature $T$.

A further insight into the physical meaning of $\mathcal{J}_{\text {can }}$ be gained from a comparison of (2.8) with (1.6b). The latter equation states that Hamilton's Principal Function $\mathcal{f}$ can be regarded as the reading of a clock whose rate is the Lagrangian $L$ which, in the absence of any potential energy, is just equal to the kinetic energy. But the temperature $T$ is just equal to (except for a proportionality constant) the kinetic energy of random thermal motion. This suggests that $\mathcal{J}$ plays a role analogous to $\&$, except that it refers to the energy of random thermal motion rather than to the macroscopic kinetic energy. 
Referring to (1.6a), we see that this analogy suggests that $\underset{m}{\nabla} \widetilde{J}$ should in some way be related to a thermal momentum flux in the fluid, i.e. the momentum associated with a heat flux. This thought is reinforced by writing $(2.12)$ in the following form:

$$
\partial p / \partial t=-m p p+v \times(\nabla \times p)
$$

where

$$
\underset{m}{p}=m \underset{m}{ }+(q / c) A+m \Delta \nabla r
$$

and

$$
P=m\left[G+h+\frac{1}{2} v^{2}+(q / m) A^{0}-\Delta \partial J / \partial t\right] .
$$

We note that $p$ has the form of a generalized canonical particle momentum in which the term $m \Delta \nabla \mathfrak{J}$ is the thermal contribution. This fact, together with the above-mentioned analogy of $\mathfrak{J}$ with $\mathcal{\&}$, suggests that the thermal function $\mathcal{J}$ could perhaps be used to describe heat flux as well as temperature (i.e. heat energy density). It is evident that (2.8) does not completely determine $\mathfrak{F}$ since it does not specify the difference in the values of $\widetilde{J}$ associated with different flow lines at a given instant of time. Because we have postulated an ideal fluid, and so are ignoring heat flux, we shall simply take the point of view that the degrees of freedom in $\widetilde{J}$ that are not specified by $(2.8)$ may be arbitrarily specified according to mathematical convenience, i.e. they may be regarded as inconsequential constants of integration. 


\section{Thermal Gauge Invariance}

Part of the indeterminacy in $\mathfrak{F}$ can be described in terms of a gauge indeterminacy that is completely analogous to what we encounter in the case of the electromagnetic scalar and vector potentials. We note from (2.13b) and (2.13c) that if we add an arbitrary function of $s$ to $\widetilde{J}$, then $p$ is increased by the gradient of some scalar function $\psi$, and $P$ is diminished by $\partial \psi / \partial t$. Thus

$$
p_{m}^{\prime}=p_{m}+\underset{m}{\nabla} \psi
$$

and

$$
\gamma^{\prime}=\gamma-\partial \psi / \partial t
$$

But if $p$ and $P$ satisfy Euler's equation (2.13a), then so do $p^{\prime}$ and $f^{\prime}$. Thus Euler's equation does not suffice to specify the gauge function $\psi$. As in the case of the electromagnetic gauge, the choice may be made on the basis of mathematical convenience. We shall later see that a particular choice suggests itself in a very natural way.

\section{Intrinsic Vorticity}

Inasmuch as the definition of vorticity for an uncharged isentropic fluid is $\frac{1}{2} \nabla \times \underset{m}{V}=$ $(\underset{m}{\nabla} \times m v) / 2 m$, a natural generalization would consist of replacing the particle momentum $m v$ with the canonical momentum $\underset{m}{p}$ defined in $(2.13 b)$. Doing this, $(2.13 a)$ becomes

$$
\partial p / \partial t=-m \nabla p+2 m \underline{m} \times \underline{m}
$$


where

$$
\omega=(\nabla \times p) / 2 m
$$

We can gain an insight into the physical significance of $\boldsymbol{\omega}$ by noting that $(2.15 \mathrm{~b})$ can be written in the following form:

$$
\Omega=\Omega_{m L}+\Omega_{\mu}+\underline{m}
$$

where

$$
\begin{aligned}
& \Omega_{m}=\frac{1}{2} \nabla \times y \\
& \Omega_{m}=-(q / 2 m c) g_{m} \\
& \Omega_{m}=\frac{1}{2}(\nabla J \times \nabla s) .
\end{aligned}
$$

$\Omega$ is the local angular velocity of the fluid. $\Omega_{L}$, whose magnitude is the Larmor frequency, represents the contribution to $\Omega$ that results from the diamagnetic response of the charged fluid to the imposition of the magnetic field $\underset{m}{B} . \Omega_{\mathrm{m}}$ is an analogous contribution to $\Omega$ that is thermal, rather than magnetic, in origin. It vanishes in the isentropic case $\left(\nabla_{\Delta} \Delta=0\right)$, and even for the weaker adiabatic requirement, the undetermined degrees of freedom of $\mathcal{J}$ can often be used to make $\Omega$ vanish. Thus $\omega_{m}$ is that part of $\Omega$ that is not actively produced either by the magnetic field or by thermal effects, but rather is the part of $\Omega$ that is intrinsic to the fluid and is to be associated with the 
initial conditions of the fluid. For this reason it will be called the intrinsic vorticity. (The definition $(2.15 \mathrm{~b})$ indicates that the name canonical vorticity would also be appropriate.) In order to distinguish between $\omega_{m}$ and $\Omega$, the latter will be referred to as the rotation, and the term vorticity will be reserved for $\underline{m}$.

The importance of $w_{m}$ arises from the fact that it, rather than $\Omega$, obeys the generalized vorticity conservation theorem, which we shall now derive.

\section{Generalized Vorticity Conservation}

Taking the curl of $(2.15 a)$, and using $(2.15 b)$, we find

$$
\partial \omega_{m} / \partial t=\underset{m}{\nabla} \times(\underline{m} \times \underset{m}{\omega})
$$

It follows directly from $(2.15 b)$ that

$$
\nabla \cdot \omega=0
$$

It is well-known that a vector which satisfies equations of the form (2.17) and (2.18) must also satisfy the following relation:

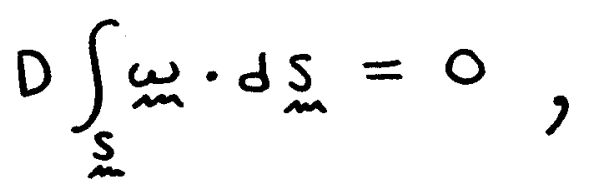

where the integration is taken over an arbitrary surface $\mathrm{S}_{\mathrm{m}}$ that is frozen into the fluid and moves with it. 
Equation (2.19) is the desired generalization of the vorticity conservation theorem.

We note that for an uncharged isentropic fluid $B=\nabla_{\Lambda}=0$, so $\underset{\mu}{w}$ reduces to $\Omega_{\mu}$ and (2.19) becomes the familiar Helmholtz (or Kelvin) theorem.

\section{VORTICITY CONSTANTS OF MOTION}

As a preliminary to deriving a more convenient formulation of the vorticity conservation theorem, we express the generalized canonical momentum $\underset{m}{p}$ in terms of three scalar functions ${ }^{(2)} S, M$, and $\phi$ :

$$
p_{m}=\nabla_{m}(m S)+M \nabla_{m} \phi
$$

where for later convenience $S$ has been multiplied by the particle mass $m$. Referring to $(2.14 \mathrm{a})$ we see that $\mathrm{mS}$ includes the indeterminate thermal gauge function. The significance of $M$ and $\phi$ becomes apparent when we substitute (3.1) into (2.15b):

$$
\omega_{m}=(2 m)^{-1}(\nabla M) \times\left(\nabla_{m} \phi\right)
$$

Thus $M$ and $\phi$ determine the intrinsic vorticity $\omega_{m}$. We must now find the equations of motion satisfied by $M$ and $\phi$. These two scalar equations will then replace the vector equation (2.17). (Note that, because $\boldsymbol{\omega}$ must be solenoidal, it can have only two degrees of freedom.)

To arrive at the desired equations of motion for $M$ and $\phi$, we substitute (3.1) into (2.15a): 


$$
\begin{aligned}
(\partial M / \partial t) \nabla \phi-(\partial \phi / \partial t) \nabla M= & -\nabla(p+m \partial S / \partial t+M \partial \phi / \partial t) \\
& +2 m \underset{m}{v} \times \underset{m}{\omega} .
\end{aligned}
$$

We now make a specific choice of thermal gauge as determined by the equation

$$
\begin{aligned}
-\partial S / \partial t & =P / m+(M / m) \partial \phi / \partial t \\
& =G+h+\frac{1}{2} v^{2}+(q / m) A^{0}-\Delta \partial J / \partial t+(M / m) \partial \phi / \partial t .
\end{aligned}
$$

For this choice of thermal gauge (3.3) becomes

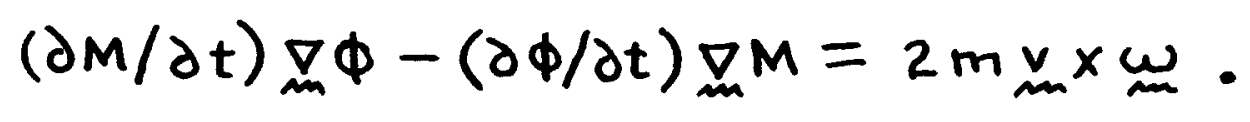

But from (3.2) we have

$$
2 m \underset{m}{x} \underset{m}{w}=\left(v_{m} \cdot \nabla \phi\right) \underset{m}{\nabla} M-\left(x_{m} \cdot \nabla M\right) \nabla_{m} \phi .
$$

Subtracting (3.6) from (3.5) we find

$$
(D M) \underset{m}{\nabla} \phi-(D \phi) \underset{m}{\nabla} M=0 .
$$

Taking the cross-product of this equation with $\underset{m}{M}$, and using (3.2) we have

$$
\text { (DM) } \underset{m}{\omega}=0 \text {. }
$$

Thus, if $\underset{w}{\omega} \neq 0$,

$$
D M=0
$$

13 
Similarly,

$$
D \phi=0
$$

If $\underset{m}{\omega}=0$, then $\underset{m}{p}$ must be the gradient of a scalar, and $M$ and $\phi$ may be set equal to arbitrary constants (or zero) with no loss of generality.

Equations (3.9) and (3.10) are the desired scalar equations of motion that describe the vorticity and replace the vector equation (2.17). These equations show that, for the choice of thermal gauge made in (3.4), $M$ and $\phi$ are constants of motion of the fluid. It is this fact that makes this choice of gauge the most natural one.

From (3.2) it is evident that the product $M \Phi$ must have the dimensions of angular momentum. With no loss of generality we may assert that $M$ has the dimensions of angular momentum and that $\phi$ is dimensionless. Thus $M$ may be regarded as an angular momentum, in some way related to the vorticity, that is conserved along a flow line. We may think of $\phi$ as the initial value at $t=0$ of one of the particle coordinates (in dimensionless form), whose memory is retained by the particle for all time.

As an illustration, consider the simple case of rigid cylindrical rotation of an uncharged fluid. The constant angular velocity is $\Omega=\Omega \mathrm{m}$ where $\underset{m}{\mathrm{~m}}$ is the unit vector along the cylindrical axis. Let $r$ be the distance from the axis and $\varphi$ the azimuthal angle. Then if

$$
M=m \Omega r^{2}
$$


and

$$
\phi=\varphi-\Omega t,
$$

it is easily verified that (3.9) and (3.10) are satisfied. It follows from (3.2) and (3.11) that

$$
\omega_{m}=(2 m)^{-1}(\nabla M) \times(\nabla \phi)=\Omega k
$$

In this case the intrinsic vorticity $\boldsymbol{w}$ and the rotation $\Omega$ are identical, and $M$ is just the particle angular momentum. For a more general vortex, however, in which the magnetic force were taken into account, we see from $(2.16 a)$ that $\Omega \neq \omega$. For such a vortex, $M$ would not be the total particle momentum, but only that part of it caused by the intrinsic vorticity, i.e. $M=m \omega r^{2}$.

Since in the present example the azimuthal angle $\varphi$ of a particle is given by $\varphi=\Omega t+\varphi_{0}$, where $\varphi_{0}$ is the particle's azimuthal coordinate at $t=0$, it is evident that $\phi=\varphi_{0}$. Thus, in this case it is the azimuthal coordinate whose initial value the particle remembers.

Finally, we note that for this example

$$
M \underset{m}{\nabla} \phi=m \Omega r \varphi_{m}
$$

where $\varphi_{1}$ is the unit vector in the azimuthal direction. Referring to (3.1), we see that

$M \underset{m}{\nabla}$ is the contribution to $\underset{m}{p}$ that is to be associated with the intrinsic vorticity $\underset{m}{\omega}$,

which in this case ${ }_{\wedge}$ just equal to the angular velocity $\Omega$. Since the right side of (3.13) 
is equal to the particle momentum $\underset{m}{\mathrm{~m}}$, which in this case is identical to $p$, we see that for this example there is no potential contribution $\nabla(m S)$ to $p$.

\section{Transition to $w_{m}=0$}

From (3.2) we note that if either $M$ or $\phi$ becomes everywhere constant, then $w$ vanishes. Moreover, because $M$ and $\phi$ are constants along each flow line, if either one becomes everywhere constant at any instant of time (i.e. assumes the same value on all flow lines), it must remain constant for all time. This corresponds to the fact that if, at any instant of time, $\underset{m}{w}=0$ in (2.17), then its time derivative also vanishes everywhere, and so $w_{m}$ must remain zero for all later time.

The use of the scalar constants of motion $M$ and $\phi$ to describe the vorticity provides an insight into the manner in which the transition from $\omega_{m} \neq 0$ to $\omega=0$ takes place. In a fluid free of small scale instabilities such a transition could never come about. If, however, the Reynolds number is sufficiently large, turbulance mixes the fluid so that the values of the constants of motion $M$ and $\phi$ (and $s$ ) on neighboring flow lines tend to equalize. This process could be pictured as a diffusion of the constants of motion from regions in which they have large values into regions in which the values are smaller. If the turbulent mixing and the time lapse since $t=0$ are large enough, the fluid will have forgotten the various values of $M$ and $\phi$ that were assigned to each of the flow lines at $t=0$. In this case, it will have made the transition to values that are constants throughout the fluid, which means that the fluid has permanently settled 
into the condition characterized by $\omega_{m}=0$. When the value of $A$ becomes everywhere constant, the fluid has made the transition from adiabatic to isentropic flow.

Even when abundant turbulant mixing occurs, however, the transition to constant values of $M, \phi$, and $A$ will not take place if an external constraint or boundary condition prevents it. For example, a rotating paddle wheel could inject vorticity (i.e. large values of $M$ and $\phi$ ) into the fluid in such a way as to compensate for the decrease in $M$ and $\phi$ caused by diffusion (i.e. turbulent mixing).

The constraint could also be geometric in nature as, for example, in the case of a fluid blowing radially outward from a central point (e.g. the solar wind). In this case as time elapses the ever increasing distance between what had been neighboring blobs of gas at $t=0$ makes mixing ever more difficult.

The above two constraints could occur in an ideal (i.e. frictionless) fluid. If we admit the possibility of friction, we have an additional mechanism that can prevent the transition to $w_{m}=0$. From (2.16a) we see that, to the extent that $\Omega_{m}$ is negligible, $\omega_{m}=0$ means that $\Omega=-q B / 2 m c$. Thus, in the case of a fully ionized plasma consisting of electron and ion gases, the two gases must rotate in opposite directions if each is individually to fulfill the condition $\underset{m}{w}=0$. But the friction between the two counterrotating gases, which is proportional to the electrical resistivity of the plasma, opposes this relative rotation and tends to make it vanish. Thus in this case friction works against the tendency of turbulent mixing to reduce $\underset{m}{\omega}$ to zero. 
These examples illustrate that in many situations the transition to the condition $\omega=0, \Delta=$ constant does not occur because of constraints of one kind or another. If, however, no such constraints are present, and if one is not interested in a transient solution that is dependent on a particular set of initial conditions, then the particular solution characterized by $\underline{\omega}=0, \Delta=$ constant has greater physical significance than any other solution that satisfies the same boundary conditions, in the sense that it represents the "steady-state" condition to which the fluid will be brought by turbulent mixing. Such "steady-state" solutions should be of particular importance in astrophysical problems in which the Reynolds number, and hence the turbulent mixing, is generally extremely large.

\section{Potential Flow}

Referring to (3.1), we note that if either $M$ or $\phi$ is a constant (or if $M$ is an arbitrary function of $\Phi$ ), the term $M \nabla \phi$ can be expressed as the gradient of a scalar, and so can be absorbed into the term $\nabla(m s)$. In such a case $\underset{m}{p}$ is just the gradient of a scalar and $\omega_{m}=0$. Such flow will be designated adiabatic potential flow. Referring to (2.13b), we see that for such flow

$$
\begin{array}{r}
\underset{m}{\nabla} S=\underset{m}{v}+(q / m c) A+\Delta \nabla_{m} \mathcal{J} . \\
\text { (Adiabatic Potential Flow) }
\end{array}
$$

If now $\Delta$ should become constant, then the term $\Delta \nabla \Im$ in (3.14) becomes the gradient of the scalar $\Delta \mathcal{J}$ which can be absorbed into the potential function $S$. Such flow will be designated isentropic potential flow. It is characterized by the condition 


$$
\nabla S=v+(q / m c) \text { A }
$$

(Isentropic Potential Flow)

In the above discussion we noted that, in the absence of any constraints that would prevent its realization, the flow characterized by the conditions $\underline{\omega}=0$ and $s=$ constant, which is just isentropic potential flow, represents the "steady-state" flow brought about by turbulent mixing. Taking the curl of (3.15), we can characterize such flow by the relation

$$
\frac{1}{2} \underset{m}{\nabla} \times \underset{m}{V}=-(q / 2 m c) \underset{m}{B}
$$

(Isentropic Potential Flow)

or $\Omega_{m}=\Omega_{L}$, i.e. the direction of the local angular velocity vector is opposed to the magnetic field vector and has the magnitude of the Larmor frequency.

It is of interest to note parenthetically that this condition could not be fulfilled by a constant-pressure charged fluid, because in this case there is no pressure gradient to help provide the necessary centripetal force, which must then be provided solely by the magnetic force. This requires that $\Omega=2 \Omega$, i.e. the angular velocity must equal the cyclotron frequency $\left(2 \Omega_{\downarrow}\right)$ rather than the Larmor frequency. Referring to $(2.16 \mathrm{a})$ we see that the condition $\Omega=2 \Omega_{m}$ (in the case of isentropic flow for which $\Omega_{\Delta}=0$ ) requires that $w_{m}=w_{m L}$. Thus constant-pressure isentropic flow of a charged fluid is characterized by the presence of an intrinsic vorticity whose magnitude is the Larmor frequency. (Note that if $\omega_{m}=-\Omega_{n}$, then $\Omega_{\mu}=0$. Thus if rotation is present $w=\Omega_{h}$, and if the rotation is zero $\underset{m}{w}=-\Omega_{m}$. In either case $|\underline{m}|=\left|\Omega_{m}\right|$.) 
IV. SCALAR FORMULATION

Adiabatic Flow

Using $(2.13 b)$ to eliminate $p$ in $(3.1)$, we obtain the following expression for $\stackrel{v}{m}:$

$$
\stackrel{v}{v}=\nabla S-(q / m c) A-\Delta \nabla_{m} \mathcal{J}+(M / m) \nabla_{m} \phi,
$$

which is the generalization of (1.1). Using (4.1) to eliminate $\frac{1}{2} \gamma^{2}$ in (3.4), we arrive at the generalization of $(1.7)$ :

$$
\begin{aligned}
-\partial S / \partial t+\Delta \partial J / \partial t-(M / m) \partial \phi / \partial t & =G+h+(\% / m) A^{0} \\
& +\frac{1}{2}[\nabla S-(q / m c) A-\Delta \nabla J+(M / m) \nabla \phi]_{m}^{2},
\end{aligned}
$$

which will be referred to as the generalized Hamilton-Jacobi (H-J) equation.

From (3.4) and (4.1) we find, making use of (3.9) and (3.10), that

$$
D S=\frac{1}{2} v^{2}-\left[G+h+(q / m c)\left(c A^{0}-y \cdot A\right)\right],
$$

which is a very natural generalization of (1.4) inasmuch as, except for the factor $\left[1-(v / c)^{2}\right]^{-1 / 2}$ which is unity in the non-relativistic limit in which we are working, $\left(c A^{0}-v \cdot A\right)$ is just the contraction of the 4-vector potential and the fluid 4-velocity, which means that the extra term in (4.3) that is missing from (1.4) is just the contribution of the electrostatic potential in the fluid rest-frame. 
The density $\rho$ can be found from the continuity equation. Using (4.1), we have

$$
\begin{aligned}
D \ln \rho & =-\nabla \cdot v \\
& =-\nabla^{2} S+(q / m c) \nabla_{m} \cdot A+\nabla \cdot(\Delta \nabla J)-m^{-1} \nabla \cdot(M \nabla \phi),
\end{aligned}
$$

which is the generalization of (1.8).

The independent variables of the problem are $S, M, \phi, \mathcal{A}$, and $\widetilde{\mathcal{S}}$. The generalized $H-J$ equation (4.2) plus the side conditions $D M=D \Phi=D s=0$ provide four of the five necessary equations. (Note that the $v$ that appears in the $D$ operator is to be either eliminated by means of (4.1), or else treated within the framework of an iteration procedure as a given vector function.)

To complete the system of equations, we need an equation for $\mathfrak{J}$. In order to derive such an equation, we must know the thermodynamic properties of the fluid. For the sake of illustration, let us consider the fluid to be a perfect gas for which the ratio of specific heats is $\gamma=c_{p} / c_{v}$. Because for a perfect gas $h=c_{p} T$, we have using

$$
h=c_{p} D \mathcal{J}
$$

(Even in the case of an arbitrary fluid, $h$ can always be expressed as a function of $T=D \mathcal{F}$ and $s$, and so is not an independent variable.) For a perfect gas

$$
A=(k / m)\left[(r-1)^{-1} \ln h-\ln \rho\right]+\text { constant }
$$


where $k$ is the Boltzmann constant. From (4.6), the continuity equation (2.2b), and the adiabatic condition $(2.10)$, we find

$$
D h=-\left[(r-1) \nabla_{m} \cdot v_{m}\right] h \text {, }
$$

which can be written in terms of $\mathfrak{J}$ by means of (4.5):

$$
D^{2} \mathcal{J}=-\left[(\gamma-1) \nabla \cdot v_{m}\right] D J
$$

This is the desired equation for $\mathcal{J}$ which completes our system of five equations for the independent variables $S, M, \Phi, A$, and $\widetilde{J}$.

In the chart given in Figure 1 a comparison is made between the Euler and the scalar formulations. In the Euler formulation we may regard the three components of $\underset{m}{v}$ as the dynamical variables, and $P$ and $\rho$ as the thermodynamic variables. The dynamical equations are the three components of the Euler equation; the equation for

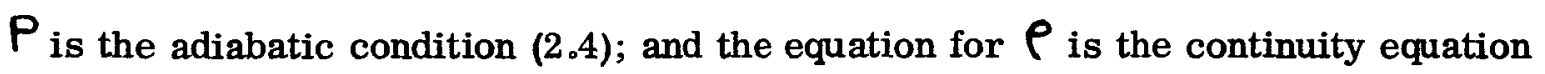
(2.2b).

In the scalar formulation $\underset{m}{V}$ is replaced by the three scalars $S, M$, and $\phi$, and the Euler equation is replaced by the $H-J$ equation and the two side-conditions $D M=D \Phi=0$. In the case of potential flow, a significant simplification occurs in the scalar formulation in that $M$ and $\Phi$ drop out. No corresponding simplification occurs in the Euler formulation. 
The thermodynamic variables in the scalar formulation are $s$ and $\mathcal{J}$ which must satisfy the equations $D_{s}=0$ and (4.8), which are roughly comparable in difficulty with the equations for $P$ and $P$ in the Euler formulation. The density $P$ does not enter into the scalar formulation at all. After the problem has been solved, and $A$ and $\mathcal{J}$ (and hence $h$ ) are known functions, $\rho$ can be found from (4.6) which, solved for $\rho$, becomes

$$
\rho=N h^{1 /(r-1)} e^{-m \Delta / k}
$$

where $\mathbf{N}$ is a normalization constant.

When the flow is isentropic rather than adiabatic, the only surviving thermodynamic variable is $h$. Thus the single equation (4.7) replaces the two equations for $A$ and $\mathcal{J}$ that were needed for adiabatic flow. The only corresponding simplification that occurs in the Euler formulation is that the algebraic equation (2.3) replaces the differential equation (2.4).

In the case of isentropic potential flow, the only surviving variables in the scalar formulation are $S$ and $h$, and the $H-J$ equation assumes the following simplified form:

$$
-\partial S / \partial t=G+h+(q / m) A^{0}+\frac{1}{2}\left[\nabla S-(q / m c) A_{m}\right]^{2} \text {. }
$$

This has exactly the form of the single-particle $H-J$ equation except for the presence of $h$.

It is obvious that the advantage of the scalar formulation is greatest in the case of isentropic potential flow. In the more general case of adiabatic non-potential flow that 
differs only slightly from isentropic potential flow, a simple perturbation solution is possible, using a solution of (4.10) as the zero-order solution. This procedure is facilitated by noting that, using $D \Phi=0, D \widetilde{S}=T$, and $(4.1),(4.2)$ can be written

$$
\begin{aligned}
-\partial S / \partial t= & G+(h-T \Delta)+(q / m) A^{0}+\frac{1}{2}[\nabla S-(q / m c) A]^{2} \\
& -\frac{1}{2}\left[\Delta \nabla_{m} J-(M / m) \nabla \phi\right]^{2},
\end{aligned}
$$

where in this form of the generalized $\mathrm{H}-\mathrm{J}$ equation it is the specific Gibbs function $g=h-T_{A}$, rather than $h$, that plays the roles of the thermal potential. The terms $-T_{\Delta}$ and $-\frac{1}{2}[\Delta \nabla \sigma-(M / m) \nabla \Phi]^{2}$ in (4.11) constitute the perturbation. Because both of these terms will be small for flow that is only slightly different from isentropic potential flow, the change in $S$ will be small, and we may linearize the term $\frac{1}{2}[\nabla S-(q / \mathrm{mc}) \mathrm{A}]^{2}$ in calculating this change in $S$.

\section{Constant-Temperature Flow}

The term constant-temperature flow will be used to designate flow that satisfies the condition

$$
D T=0
$$

which replaces the adiabatic condition (2.10). Flow satisfying the stronger condition, $T=$ constant, will be designated isothermal flow.

The derivation of the scalar formulation in the case of constant-temperature flow is completely analogous to that for adiabatic flow. We can eliminate the density in 
Euler's equation (2.1) by means of the thermodynamic identity

$$
e^{-1} \nabla_{m} P=m \nabla_{m} g+m \Delta \nabla_{m} T
$$

where

$$
g=h-T A
$$

is the specific Gibbs function. In order to transform the term $m \Delta \underset{m}{\nabla} T^{\text {thus introduced }}$ into Euler's equation, we define a function $\&$ by means of the equation

$$
D \&=s
$$

By means of an identity analogous to (2.9), Euler's equation can be written in the form

$$
\partial p_{m} / \partial t=-m \nabla_{m} p+v_{m} \times\left(\nabla_{m} \times p\right)
$$

where now, in the constant-temperature case,

$$
p_{m}=m \underset{m}{v}+(q / c) A-m T \nabla_{m} L
$$

and

$$
p=m\left[G+g+\frac{1}{2} v^{2}+(q / m) A^{0}+T \partial \ell / \partial t\right] .
$$

Equations (3.1) and (3.2) are valid for constant-temperature flow as well as for adiabatic . flow. Thus we see that any adiabatic flow equation can be converted into the corresponding 
constant-temperature flow equation by $\operatorname{replacing}(h, s, T$, and $\mathfrak{J})$ by $(g,-T, s$, and \&) respectively and making no changes in $S, M, \Phi$ or $\underset{m}{\omega}$.

The generalized $\mathrm{H}-\mathrm{J}$ equation for constant-temperature flow is

$$
\begin{aligned}
-\partial S / \partial t-T \partial \& / \partial t & -(M / m) \partial \phi / \partial t=G+g+(q / m) A^{0} \\
& +\frac{1}{2}[\nabla S-(q / m c) A+T \nabla \&+(M / m) \nabla \phi]_{m}^{2}
\end{aligned}
$$

This equation must be augmented by the three side conditions $D T=D M=D \phi=0$. To complete the system of equations we need an equation for $\&$ analogous to (4.8). This equation, like (4.8), will depend on the thermodynamic properties of the fluid. As before, we shall consider a perfect gas. Since in this case $h=c_{p} \top$, we have

$$
g=h-T \Delta=T\left(C_{p}-D \&\right)
$$

and so

$$
D g=-T D^{2} \&
$$

where we have used (4.12). It is possible to arrive at an independent expression for Dg from the thermodynamic identity

$$
e^{-1} D P=m D g+m A D T=m D g,
$$

where in the second step we have again used (4.12). Since for a perfect gas $P=k e T$, (4.20) becomes 


$$
D g=(k T / m) D \ln \rho=-(k T / m) D \cdot m,
$$

where in the second step we have used the continuity equation (2.2b). Finally, from (4.19) and (4.21) we have

$$
D^{2} d=(k / m) \underset{m}{\nabla} \cdot v_{m},
$$

which is the desired equation for $\mathcal{L}$ that is analogous to (4.8). Thus we have completed our system of equations for the three dynamical variables $S, M$, and $\phi$ and the two thermodynamic variables $T$ and $\ell$. The density $P$ is not one of the simultaneous variables, but once the problem has been solved, $\rho$ can be found from

$$
\rho=N T^{1 /(\gamma-1)} e^{-m \Delta / k}
$$

which is just $(4.9)$ with the factor $C_{p}^{1 /(\gamma-1)}$ absorbed into the normalization constant N. The velocity $\underset{m}{\checkmark}$ can be found by substituting (3.1) (which remains valid for constanttemperature flow) into $(4.16 \mathrm{~b})$ :

$$
\underset{m}{v}=\nabla_{m} S-(q / m c) A_{m}+T \underset{m}{\nabla} Q+(M / m) \nabla_{m} \phi
$$

The form of the constant-temperature $\mathrm{H}-J$ equation that is analogous to (4.11) is

$$
\begin{aligned}
-\partial S / \partial t= & G+h+(q / m) A^{0}+\frac{1}{2}[\nabla S-(q / m c) A]^{2} \\
& -\frac{1}{2}\left[T \nabla \ell+(M / m) \nabla_{m} \phi\right]^{2}
\end{aligned}
$$


where now $h=g+T s$ plays the role of the thermal potential. This fact is especially favorable in the case of a perfect gas since then $h=c_{p} T$ and it is not necessary to use the more complicated relation (4.18) to express the thermal potential (which in the case of (4.17) is $g$ ) in terms of the thermodynamic variables $T$ and $\&$. For a perturbation calculation for nearly isothermal potential flow, however, we would write $g+T A$ in (4.25) instead of $h$, and would regard $T_{\infty}-\frac{1}{2}[T \nabla \&+(\mathrm{M} / \mathrm{m}) \nabla \phi]^{2}$ as the perturbation.

For isothermal flow ( $T=$ constant) the terms involving $\&$ in (4.17) can be absorbed into the terms involving $S$ simply by replacing $S$ with the new flow potential $S^{\prime}=S+T \&$.

The isothermal $\mathrm{H}-\mathrm{J}$ equation can also be derived directly from the adiabatic equation (4.2) simply by specifying that $\widetilde{S}=T_{c} t$ where $T_{c}$ is the constant temperature. Then $\partial \widetilde{S} / \partial t=T_{c}$ and $\nabla \widetilde{S}=0$, and so (4.2) becomes

$$
\begin{aligned}
-\partial S / \partial t-(M / m) \partial \phi / \partial t & =G+g+(q / m) A^{0} \\
& +\frac{1}{2}[\nabla S-(q / m c) A+(M / m) \nabla \phi]^{2} .
\end{aligned}
$$

(Similarly, the isentropic $\mathrm{H}-\mathrm{J}$ equation can be derived from (4.17) by specifying $\&=\Delta_{c} t$ where $\Delta_{c}$ is the constant specific entropy.) Using $D \Phi=0$ and the expression (4.24) for $\underset{m}{v}$ (without the term $T \underset{m}{\nabla} \&$ which has been absorbed into the term $\underset{m}{\nabla} S$ ), we arrive at the following alternative form of (4.26):

$-\partial S / \partial t=G+g+(q / m) A^{0}+\frac{1}{2}[\nabla S-(q / m c) A]^{2}-\frac{1}{2}[(M / m) \nabla \Phi]^{2}$.

28 
In the case of isothermal flow, the only thermodynamic variable is $g$, so we would use (4.21) as our thermodynamic equation instead of (4.12) and (4.22). The most convenient expression for $\rho$ is found by substituting $A=c_{p}-g / T$ (from (4.18)) into (4.23) yielding

$$
\rho=N e^{m g / k T_{c}} \text {. }
$$

Barotropic Fluid

In the case of a barotropic fluid there exists a thermal potential function $\eta=\eta(e)$ that is a function of the density alone such that

$$
e^{-1} \nabla_{m} P=\nabla_{m} \eta
$$

This relation can be used to eliminate $\rho$ from Euler's equation $(2.1)$. The derivation of the scalar formulation is identical to that for adiabatic flow except that $h$ must be replaced by $\eta$ and the terms involving $T$, $s$, and $\widetilde{J}$ do not appear. Thus the scalar formalism is identical to that for isentropic flow except for the replacement of $h$ by $\eta$. The independent variables are $S, M, \Phi$ and $\eta$. The three dynamical equations are the $H-J$ equation and the conditions $D M=D \phi=0$. The thermodynamic equation for $\eta$ is derived by solving the relation $\eta=\eta(\rho)$ for $\rho$ in terms of $\eta$, and then substituting this into the continuity equation $D \ln \rho=-\nabla \cdot v \quad$ to arrive at the desired equation for $\eta$. 


\section{DISCUSSION}

The scalar formulation has an obvious advantage over the Euler formulation in the case of potential flow, since two of the three dynamical variables ( $M$ and $\phi$ ) drop out, whereas in the Euler formulation it is necessary to retain three variables, namely the three components of $\underset{m}{\mathrm{r}}$. In simple one-dimensional problems this advantage vanishes since $y$ has only one degree of freedom, but for three-dimensional problems it can be very significant.

Even in the case of non-potential flow, the fact that two of the three dynamical variables in the scalar formulation, $M$ and $\Phi$, are constants of motion should make the fluid behavior easier to visualize and perhaps easier to calculate.

The scalar formulation must always be applied within the framework of a two-fluid picture of the plasma, i.e. the dynamical equations of the ion and electron gases must be worked out individually. (A third, uncharged fluid can be added if necessary.) This procedure is more exact than the usual perfectly-conducting-single-fluid model in that the inertial effects of the electric current (essentially the electron flux) are taken into account. Because of the assumption that the electron and ion gases are ideal, however, we are neglecting the dissipative part of the plasma resistivity.

In the Euler formulation the intrinsic vorticity $\underset{m}{\omega}$ is never brought into the open since it is regarded merely as a certain differential function of the components of $\underset{m}{v}$, which are the basic variables. In keeping with this approach, all functions of the fluid 
flow, such as current density and frictional forces, are expressed as functions of $\underset{m}{\mathrm{v}}$.

In the scalar formulation $w_{m}$ (via the scalars $M$ and $\phi$ ) plays a central role, and $\underset{m}{v}$ is reduced to the status of a certain differential function of $S, M$, and $\Phi$. In keeping with this point of view, it would be natural to express such things as current density and frictional forces as functions of $S, M$, and $\phi$. Such a procedure might suggest some physically meaningful approximations that would simplify the overall problem. 
Footnotes

1. See, for example, L. D. Landau and E. M. Lifshitz, "Fluid Mechanics" (AddisonWesley Publ. Co., Reading, Mass., 1959), p. 19.

2. Such a representation is always possible for a continuously differentiable vector function. See, for example, L. Brand, "Vector and Tensor Analysis" (Wiley \& Sons, New York, 1947), p. 230.

The introduction of the scalars S, M, and in equation (3.1) represents a generalization of a procedure first used by Clebsch (Crelle's Journal, 24 (1857) and 56 (1859)) for an uncharged barotropic fluid. This procedure is summarized by H. Lamb, "Hydrodynamics" (Cambridge University Press, Cambridge, 1932), 6th ed., p. 248, and by H. Bateman, "Pariial Differential Equations of Mathematical Physics" (Cambridge University Press, Cambridge, 1932), p. 164. 


\section{Figure Caption}

Fig. 1. Comparison of Euler and Scalar Formulations. 


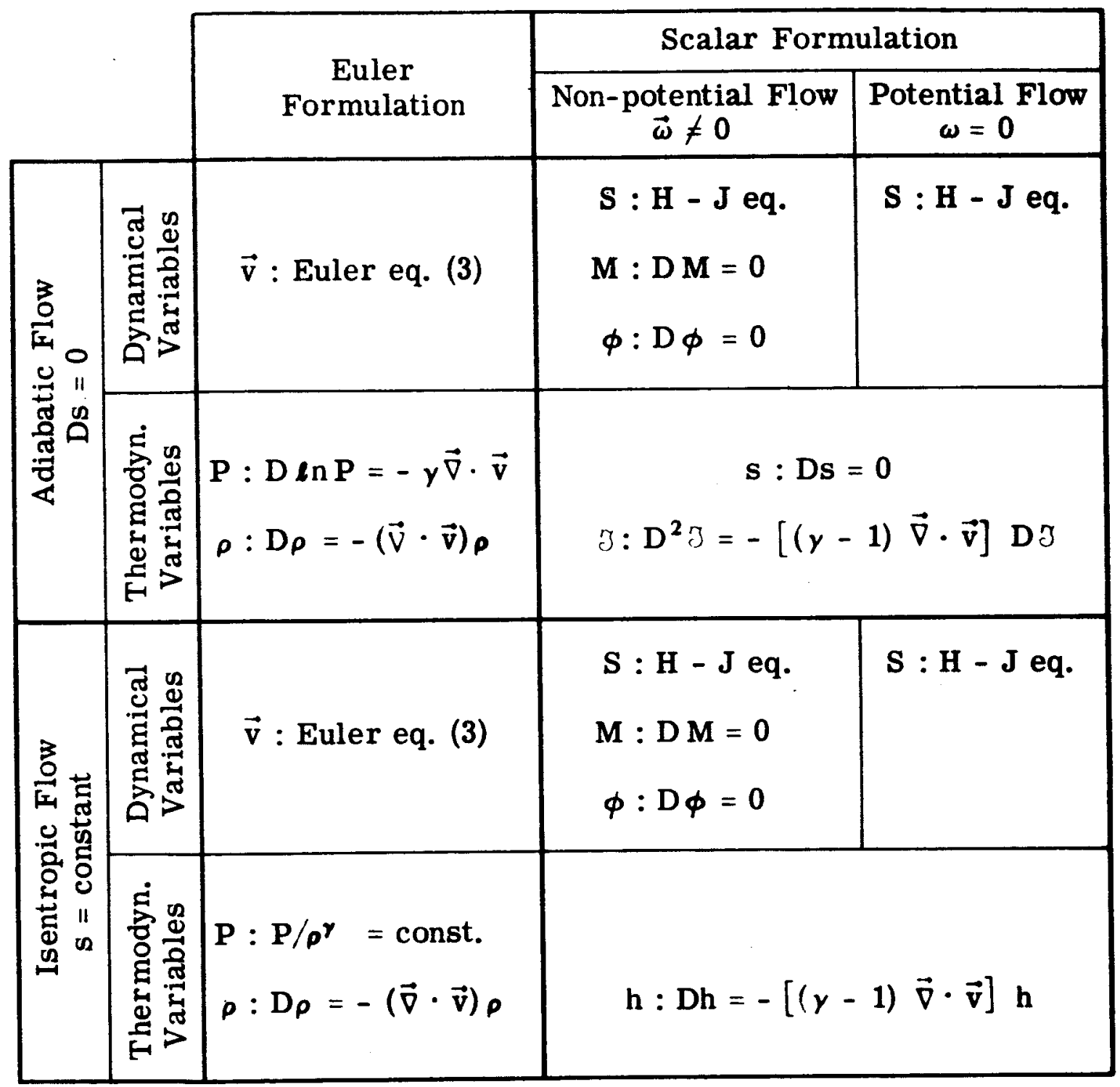

